\title{
Three Dimensional Urban Characterization by IFSAR Measurements
}

\author{
P. Gamba(*), B. Houshmand ${ }^{(1)}$ \\ (*)Dipartimento di Elettronica, Università di Pavia, Via Ferrata, 1, I-27100 Pavia, Italy \\ (†) Jet Propulsion Laboratory, 4800 Oak Grove Drive, Pasadena, CA
}

Abstract - In this paper a machine vision approach is applied to IFSAR data to extract the most relevant built structures in a dense urban environment. The algorithm tries to cluster primitives (line segments) into more complex surfaces (planes) to approximate the $3 \mathrm{D}$ shape of these objects. Very interesting results starting from TOPSAR data recorded over S. Monica are presented.

\section{INTRODUCTION}

The urban environments, with their complex structure composed of buildings of different kinds and shapes, small and/or large green areas and infrastructures have constantly been a challenge for remote sensing analysts. Despite the large number of works on the interpretation of urban images acquired by different sensors, from the classic cameras to Synthetic Aperture Radars (SAR) [1], from multispectral to hyperspectral sensors (like AVIRIS), a large amount of information is still hidden in the raw data. In particular, very few papers are devoted to the use of Interferometric SAR (IFSAR) measurements for urban image analysis: one of them is [2], where IFSAR and AVIRIS data are merged to better distinguish buildings from green areas. Indeed, the 3D measurements obtained by IFSAR may be extremely useful for extracting the complete topography of a urban environment as well as for gathering more insight on particular structures.

Analysis of the IFSAR terrain elevation data in urban areas are usually difficult due to the insufficient spatial resolution, multiple scattering due to the building geometries, and layover effects, in addition to the intrinsic IFSAR system level noise. Therefore, there is still a strong need to evaluate which type of information is available from these data and to what extent it is possible to extract them. The resolution problem is being increasingly resolved by the new generation of radar sensors operational in the near future [3]: the goal of these systems is to provide a 1-meter level spatial resolution, which therefore can resolve many of the objects present in an urban environment. As for the second problem, instead, we found very interesting to apply to the original remote sensing images some suitable machine vision approaches. Indeed, even if developed for very different situations, these procedures are of invaluable utility when used in this context.

The paper is organized as follows: Section 2 presents the building extraction algorithm, Section 3 shows the

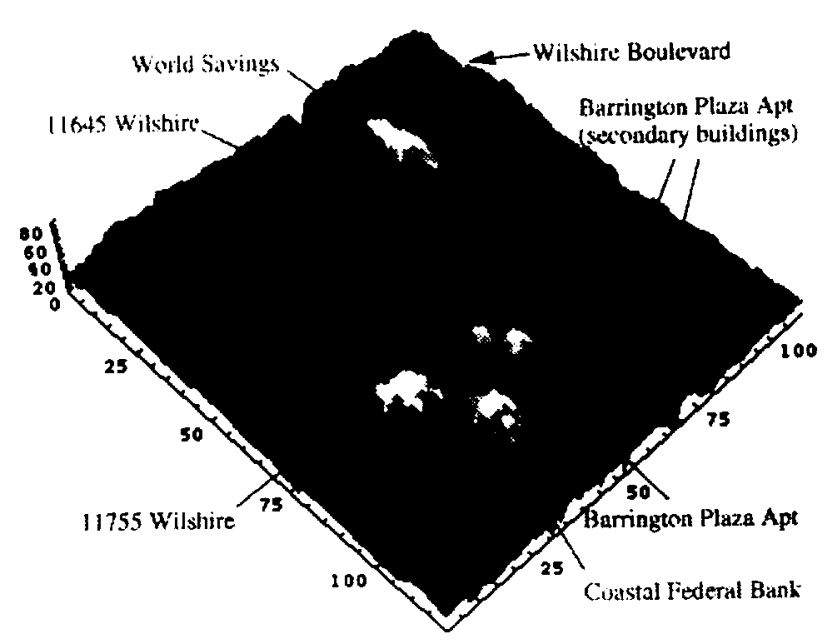

Figure 1: A range image of a part of Wilshire Boulevard, Santa Monica derived from TOPSAR interferometric measurements.

experimental results, while in Section 4 these results are discussed and the future lines of research are expressed.

\section{THE BUILDING EXTRACTION ALGORITHM}

In this work we focus on the task to extract information on urban structures from high resolution IFSAR data: specifically, we want to automate the detection of the height and shape of the buildings present in a given area. To this aim, we apply to the original data a machine vision segmentation algorithm able to exploit their resolution, while maintaining at the same time a high robustness to noise. In particular, the criteria applied to segment the raw data are geometric ones, involving the principle of plane-fitting (i.e. to find the plane which better approximates a given surface): in our situation this approach corresponds to look for the building roofs. To this aim, the simplest algorithm could be an iterative region growing approach, that is, we may start from randomly chosen pixels and examine all the adjacent one; if one pixel is near to the sample in the $3 \mathrm{D}$ space, it is added. This idea can be further improved by the algorithm outlined in [4]: in this approach the primitives of segmentation are the scan lines 


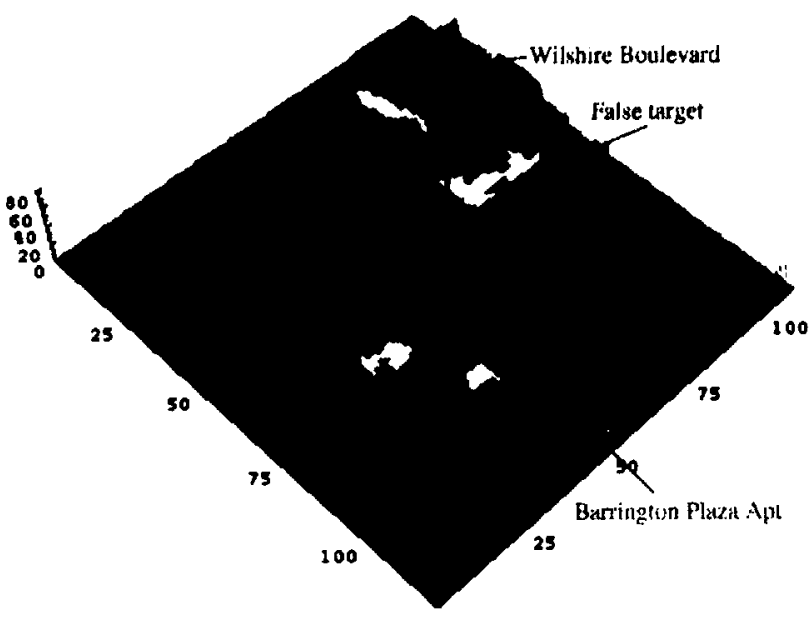

Figure 2: Classification results for the area in fig. 1 by means of the building extraction algorithm outlined in the text: note the regularization of the profile of the foreground buildings.

(lines of the image), and they are aggregated in planes by considering some suitable geometric properties.

The algorithm starts from primitives of segmentation that are the scan lines (lines of the image), in order to save cpu time. Then it works by means of three processing steps, that here we simply recall.

1. First, we group the pixels of each line into segment according to the following geometric criterion: a line is broken in the point furthest from the chord joining the extremes, provided that this distance is greater than a given threshold. This procedure is iterated until it is possible to find a breakpoint. Each segment found is then recorded in a double-linked list, with pointers to its neighbors (i.e. adjacent segments). The threshold is found by considering the local noise variance, computed on a $3 \times 3$ window around each pixel $p_{i j}$ as the mean square difference between the data and the best fitting plane

$\sigma_{n}^{(i j)}=\frac{\left[\sum_{k, l=-1}^{1}\left(p_{k+i, l+j}-a^{(i j)} k-b^{(i j)} l-c^{(i j)}\right)^{2}\right]^{\frac{1}{2}}}{9}$

2. The second step consists in finding the seeds for the planar regions that we want to use to characterize the original image. Each seed is formed by three adjacent segments (longer than a given threshold) each belonging to a different scan line. The optimal seed is chosen as the one nearest to the ideal condition of three segments belonging to the same planar surface. In this case the segment threshold is set as small as possible with respect to the physical characteristics of the object and the resolution of the image.

Moreover, the optimal seed is found by considering a similarity measure based on the cosine of the angle between each couple of segments (see [4], eq. (11)).

3. Next, an iterative region growing is performed. All the segments adjacent to the ones of the seed are examined: if a segment is close enough (with respect to both its slope and intercept) to the plane which better approximates the seeds, it is added to the region. This process is iterated (considering the new region), until no more expansion is possible, and further iterated on other seeds until the images is divided in planes.

We must note that, as said before, not all the pixels belong to a plane at the end of the procedure: points affected by large noise, or regions where no actually planar surface is observable are not aggregated.

Eventually, the best-fitting-plane for each region is approximated with an horizontal one, for a first, imprecise simulation of the building roofs.

\section{EXPERIMENTAL RESULTS}

The interferometric SAR image used in this research covers a portion of Santa Monica, in the metropolitan area of Los Angeles (see fig. 1). It is an IFSAR range image, that is to say an array of numbers representing the surface elevation with respect to a reference plane; so, this image already gives us the three-dimensional profile of the urban surface. The data were obtained with the TOPSAR system, operated by NASA/JPL and mounted on a DC8 plane. Ground truth was provided by a field recognition of the buildings, and the measurement, as accurate as possible, of their footprints and heights.

Fig. 2 shows that from these data the most relevant built structures have been individuated and extracted. The importance of this result is twofold. First, each of the buildings is reconstructed and most of the noise or shadowing/layover effect has been eliminated. Second, the extracted structure are completely isolated one from the others, allowing some sort of rasterization of the original image. Moreover, by grouping the range values into consistent, structures, we may study their distribution, to qualify the clustering results with respect to the approximate shape that we compute for each building. In fig. 3 a single building, together with a photo from the ground is shown to provide a qualitative assessment of the proposed approach.

From a quantitative point of view, Table 1 compares the heights (in meters) of the tallest buildings in the studied area with those measured. The accuracy is almost within 

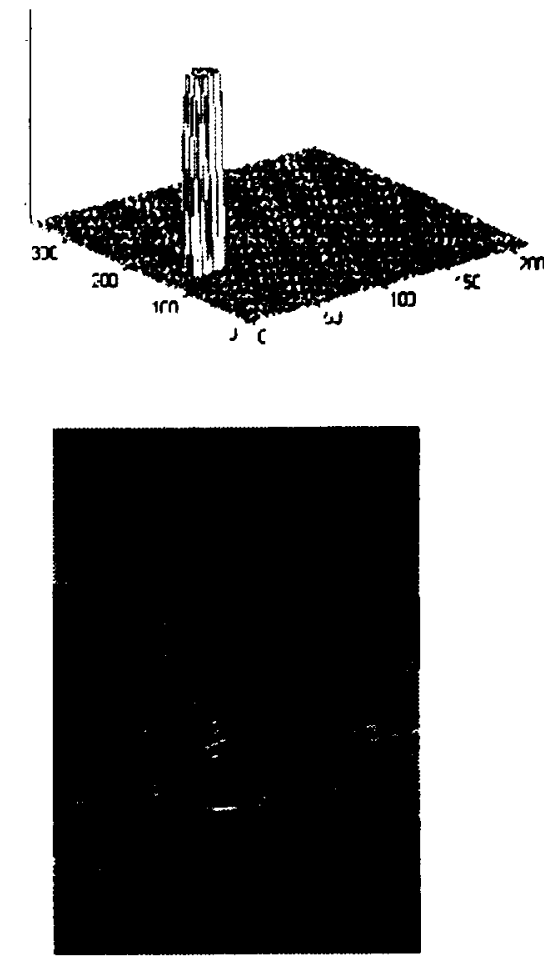

Figure 3: An example of extracted building: its 3D profile compared with a photo.

the precision of the TOPSAR sensor $( \pm 2.5 \mathrm{~m})$, revealing that the inaccuracies introduced by the grouping algorithm are negligible. Worst results are obtained, as it is easy to see in fig. 2, considering the areas of the buildings. We found that they are heavily underestimated, but also some strange situations occur. For instance, the layover effect on the farthest building in fig. 1 has been aggregated in a very large object that widely overestimate the building that is hidden in it. Furthermore, the two very small buildings indicated are indeed a single one, erroneously split both due to the radar effects and problems in the detection algorithm.

Indeed, the idea to take as primitives the image line segments introduce a privileged direction in the segmentation procedure. This choice has no influence on the results when the surfaces to be retrieved are large with respect to the starting segment primitives, as in the original application [4]. In our IFSAR image, instead, when three or four segments a few pixels long constitute a building, this direction must be carefully chosen. Therefore, if the environment to be analyzed (like in almost any urban situation) presents object with different orientations, the detection and reconstruction accuracy may be a decreasing function of the angle between the segmentation and the building direction.
Table 1: Actual and measured heights of the buildings extracted (mean error $=2.2 \mathrm{~m}, \sigma=4.9 \mathrm{~m}$ ).

\begin{tabular}{|l|c|c|c|}
\hline building & height & IFSAR height & error \\
\hline \hline Coastal Federal Bank & 81 & 86 & -5 \\
World Savings & 110 & 99 & -11 \\
11755 Wilshire & 98 & 99 & +1 \\
Barrington Plaza & 74 & 71 & -3 \\
11645 Wilshire & 45 & 49 & +4 \\
\hline
\end{tabular}

\section{CONCLUSIONS}

This work shows that it is possible to extract buildings from TOPSAR data by means of a suitably changed machine vision approach. The algorithm was applied to the detection of the major structures in a part of Wilshire Boulevard, S. Monica, with excellent results with respect to the retrieved heights, and some underestimate with respect to their footprints.

Future developments could be pre-processing algorithms to eliminate as much as possible the multiple scattering effects on the radar backscattered signal, before applying the clustering step. Indeed, many of this erroneous measures may be discarded by the detection procedure, but too large areas may also provide false targets, even of large dimensions. Moreover, a more refined version of the same algorithm, based on a direct plane fitting is under development.

\section{REFERENCES}

[1] F.M. Henderson and Z.G. Xia, "SAR applications in human settlement detection, population estimation and urban land use pattern analysis: a status report," IEEE Trans. on Geoscience and Remote Sensing, Vol. 35, No. 1, pp. 79-85, 1997.

[2] G.F. Hepner, B. Houshmand, I. Kulikov, and N. Bryant, "Investigation of the potential for the integration of AVIRIS and IFSAR for urban analysis," Photogrammetric Eng. Remote Sensing. Vol. 64, No. 8, pp. $512-520,1998$.

[3] M. Coltelli, G. Fornaro, G. Franceschetti, R. Lanari, A. Moreira, E. Sansoti, R. Scheiber, M. Tesauro, T.I Stein, "Results of the Mt. Etna interferometric ESAR campaign," Proc. of IGARSS'97, Vol. IV, pp. 1554-1556, Singapore, Aug. 1997.

[4] X. Jiang and H. Bunke, "Fast segmentation of range images into planar regions by scan line grouping," Machine Vision and Applications, No. 7, pp. 115-122, 1994. 